\title{
DESENVOLVIMENTO INICIAL DE MUDAS DE Euterpe ssp. APÓS A APLICAÇÃO DE HERBICIDAS ${ }^{1}$
}

\author{
JULIANA ROBERTA GOBI QUEIROZ², ANTONIO CARLOS DA SILVA JUNIOR ${ }^{3}$, \\ MARIA RENATA ROCHA PEREIRA ${ }^{4}$, DAGOBERTO MARTINS
}

RESUMO - O conhecimento sobre a aplicação de herbicidas em palmeiras frutíferas é quase inexistente. Assim, esta pesquisa teve como objetivo avaliar o desenvolvimento inicial de mudas de Euterpe oleraceae e Euterpe edulis após a aplicação de herbicidas.Foram conduzidos estudos em duas épocas (fevereiro de 2013 e janeiro de 2014) para cada espécie, no delineamento experimental inteiramente casualizado, com quatro repetições. Os tratamentos testados foram: fluazifop-p-butyl (93,8 $\left.\mathrm{g} \mathrm{ha}^{-1}\right)$; sethoxydim (184 $\left.\mathrm{g} \mathrm{ha}^{-1}\right)$; quizalofop-p-ethyl $\left(75 \mathrm{~g} \mathrm{ha}^{-1}\right)$, (clethodim + fenoxaprop-p-ethyl) $\left(50+50 \mathrm{~g} \mathrm{ha}^{-1}\right)$; fomesafen $\left(225 \mathrm{~g} \mathrm{ha}^{-1}\right)$; lactofen (168 $\left.\mathrm{g} \mathrm{ha}^{-1}\right)$; nicosulfuron $\left(50 \mathrm{~g} \mathrm{ha}^{-1}\right)$; MSMA (1.422 $\left.\mathrm{g} \mathrm{ha}^{-1}\right)$, além de uma testemunha sem aplicação de herbicida. A pulverização foi realizada sobre as mudas que apresentavam altura entre 45 e $50 \mathrm{~cm}$. Foram realizadas avaliações visuais de fitotoxidade, altura das plantas aos $7 ; 14 ; 21 ; 28 ; 35 ; 42$ e 49 dias após a aplicação (DAA)e ao final a massa seca da parte aérea.Visualmente, todos os herbicidas testados foram seletivos às duas espécies de palmeiras estudadas e quando ocorreram sintomas de injúrias(máximo 14\%), estes dissiparam-se aos 49 DAA. Ao analisar-se a altura e o acúmulo de massa seca das plantas, observou-se que a palmeira E. oleracea foi mais tolerante aos herbicidas que a E. edulis. Todos os herbicidas testados podem ser recomendados para E. oleracea. Quanto à E. edulis, apenas os herbicidas sethoxydim, nicosulfuron e a mistura de clethodim + fenoxaprop-p-ethyl não afetaram o desenvolvimento inicial das mudas nos dois anos de estudos.

Termos para indexação: açaí, juçara, palmiteiro, fitotoxidade.

\section{HERBICIDE SELECTIVITY IN Euterpe spp.}

ABSTRACT - Knowledge regarding the use of herbicides in palms fruit is almost nonexistent. Thus, the objective of this work was to evaluate the initial development of seedlings on Euterpeoleraceae and Euterpeedulis. Studies were conducted in two seasons (February 2013 and January 2014) for each species in a completely randomized design with four replications. The treatments tested were: fluazifop-p-butyl (93,8 $\left.\mathrm{g} \mathrm{ha}^{-1}\right)$, sethoxydim $\left(184 \mathrm{~g} \mathrm{ha}^{-1}\right)$, quizalofop-p-ethyl $\left(75 \mathrm{~g} \mathrm{ha}^{-1}\right)$, (clethodim + fenoxaprop-p-ethyl) $(50+50$ $\left.\mathrm{g} \mathrm{ha}^{-1}\right)$, fomesafen $\left(225 \mathrm{~g} \mathrm{ha}^{-1}\right)$, lactofen $\left(168 \mathrm{~g} \mathrm{ha}^{-1}\right)$, nicosulfuron $\left(50 \mathrm{~g} \mathrm{ha}^{-1}\right)$, MSMA $\left(1.422 \mathrm{~g} \mathrm{ha}^{-1}\right)$, and a control without herbicide. Spraying was performed over seedlings with $45-50 \mathrm{~cm}$ tall. Visual evaluations of phytotoxicity, plant height at 7, 14, 21, 28, 35, 42 e 49 days after spraying (DAS) and at the end of the dry mass of shoot.Visual observations showed that all herbicides were selective to the two palm species studied. Some visual injury symptoms eventually noticed (maximum 14\%), dissipated of these occurred over time 49 DAS. Analysis of the height and dry mass accumulation of plants showed that E. oleracea palm presented more tolerant to herbicides when compared to E. edulis.All the herbicides can be recommended to the $E$. oleracea. As to E.edulis, only the herbicidessethoxydim $\left(184 \mathrm{~g} \mathrm{ha}^{-1}\right)$, nicosulfuron $\left(50 \mathrm{~g} \mathrm{ha}^{-1}\right)$ and mixing clethodim + fenoxaprop-p-ethyl $\left(50+50 \mathrm{~g} \mathrm{ha}^{-1}\right)$ they did not affect the initial development of seedlings in the two years of study.

Index terms: açaí, juçara, palm heart, phytotoxicity.

\footnotetext{
${ }^{1}$ (Trabalho 288-14). Recebido em: 14-11-2014. Aceito para publicação em: 08-06-2015.

${ }^{2}$ Eng. Agr., Ma., Doutoranda em Agronomia, Unesp-FCA, Botucatu-SP. E-mail: jrgqueiroz@fca.unesp.br;

${ }^{3}$ Eng. Agr.,Me., Doutorando em Produção Vegetal, Unesp-FCAV, Jaboticabal-SP. E-mail: acsjr agro@hotmail.com;

${ }^{4}$ Eng. Florestal, Profa. Dra., Fatec, Curso de Tecnologia Silvicultura, Capão Bonito-SP. E-mail: mariarenatarp@hotmail.com;

${ }^{5}$ Prof. Livre-Docente do Departamento de Produção Vegetal, Unesp-FCAV, Jaboticabal-SP. E-mail: dmartins@fcav.unesp.br
} 


\section{INTRODUÇÃO}

Além dos palmitos das palmeiras do gênero Euterpe, obtém-se também uma polpa que é extraída de seus frutos, conhecida como açaí. Na região Norte do Brasil, esse fruto é comumente colhido da Euterpe oleracea Mart., e também da E. precatoria Mart., e nas regiões Sul e Sudeste, o açaí pode ser obtido a partir dos frutos da palmeira juçara (MACFADDEN, 2005). A polpa de açaí faz parte de uma cadeia produtiva sólida e com mercado garantido (SILVA et al., 2006), devido ao crescente interesse internacional por frutos exóticos, produzidos por comunidades em sistemas agroflorestais, por produtos naturais saudáveis, mas também pela maior visibilidade e disponibilidade nos mercados (VIEIRA et al., 2007; SCHRECKINGER et al., 2010).

A expansão do cultivo de Euterpe spp. para diversas regiões brasileiras, a execução de plantios ao longo do ano, a alta demanda por mudas, bem como o manejo inadequado da cultura têm aumentado a preocupação, por parte dos produtores, com as plantas daninhas, principalmente na fase de produção de mudas (SANTOS et al., 2007). Deste modo, vários trabalhos têm estudado estratégias e procedimentos que possibilitem a produção de palmeiras frutíferas em escala comercial de maneira mais eficiente, possibilitando a rápida formação do pomar (MARTINS et al., 2009ab; RAMOS et al., 2011). Quanto mais tempo a planta permanecer nos estádios iniciais de desenvolvimento, mais vulnerável estará às condições adversas do meio (MARTINS et al., 2009a; RAMOS et al., 2011; RIZZARDI; WANDSCHEER, 2014), como a competição exercida entre as plantas daninhas e a cultura por água, luz e nutrientes (FREITAS et al., 2007; BACHEGA et al., 2013).

De acordo com Gunathilake et al. (1993), a competição de plantas daninhas com o coqueiro (Cocos nucifera L.) pode resultar em redução de 18 a $20 \%$ na produtividade. Também em viveiros de palmeiras, o controle de plantas daninhas tem sido um problema, pois é realizado por meio de mondas periódicas, o que tem onerado o custo da muda e nem sempre é efetivo para o controle da comunidade infestante (ROMANI et al., 2010).

Vieira et al. (2007) relatam que o controle de plantas daninhas na cultura do açaí é realizado mais frequentemente por meio de capinas e roçagem, a cada dois meses, durante todo o seu ciclo, visando a minimizar a competição com estas plantas. Segundo estes pesquisadores, o uso de herbicidas em jato dirigido foi verificado em $69 \%$ das propriedades em sistema agroflorestal com glyphosate e amônio- glufosinato.

Nas últimas décadas, inúmeros herbicidas foram introduzidos no manejo de plantas daninhas visando ao controle seletivo destas espécies. No entanto, possíveis efeitos tóxicos desses produtos ainda não foram avaliados de forma satisfatória para várias espécies (MARTINS et al., 2007), inclusive frutíferas de interesse comercial no Brasil, o que limita seu uso nos pomares. A seletividade dos herbicidas baseia-se na capacidade da planta em metabolizar rapidamente o composto químico pulverizado, formando compostos não fitotóxicos (MARTINS et al., 2007; SOUZA et al., 2014).

Os estudos de seletividade de herbicidas em espécies perenes costumam ser realizados inicialmente sobre mudas, pois plantas resistentes nas fases iniciais de desenvolvimento em viveiro podem ser também na etapa adulta do pomar no campo (BRANCALION et al, 2009; PEREIRA et al., 2011).

$\mathrm{Na}$ implantação de áreas de produção de culturas com crescimento lento, como as do gênero Euterpe ssp., faz-se necessário ter o conhecimento e a identificação de herbicidas seletivos à cultura para que se tenha uma ferramenta a mais no manejo de plantas daninhas.

Assim, este estudo teve como objetivo avaliar o desenvolvimento inicial de mudas de palmeira-açaí (E. oleracea) e palmeira-juçara (E. edulis) quando submetidas à aplicação de herbicidas com potencial uso em áreas de produção.

\section{MATERIAL E MÉTODOS}

O estudo foi instalado e conduzido na FCA/UNESP, Câmpus de Botucatu-SP, em um delineamento experimental inteiramente casualizado, com nove tratamentos e quatro repetições, em dois anos consecutivos, fevereiro de 2013 e janeiro de 2014. A aplicação foi realizada sobre mudas comerciais $(45$ a $50 \mathrm{~cm})$ da palmeira-açaí $(E$. oleraceae) e palmeira-juçara (E. edulis) obtidas a cada ano em viveiros de produção de mudas. As mudas foram transplantadas para vasos plásticos, com capacidade de 3,1 L, preenchidos com solo classificado como Latossolo Vermelho-Amarelo (EMBRAPA, 2006), e a adubação foi realizada com base na análise de solo (AGUIAR et al., 2014). Os tratamentos e as doses testadas estão apresentados na Tabela 1.

Para a aplicação dos herbicidas, foi utilizado um pulverizador costal pressurizado a $\mathrm{CO}_{2}$, munido de pontas de jato plano Teejet XR 11002VS, espaçados entre si de $50 \mathrm{~cm}$, a uma pressão constante de trabalho (200 $\mathrm{kPa})$, o que proporcionou um volume 
de calda de $200 \mathrm{~L} \mathrm{ha}^{-1}$. As características ambientais por ocasião da aplicação foram: temperatura de $25^{\circ} \mathrm{C}$ e UR de $76 \%$ no primeiro ano, e de $27^{\circ} \mathrm{C}$ e UR de $70 \%$ no segundo ano.

Foram realizadas avaliações visuais de fitotoxidade aos $7 ; 14 ; 21 ; 28 ; 35 ; 42$ e 49 dias após a aplicação (DAA), por meio de uma escala de notas, na qual, ' 0 ' correspondeu a nenhuma injúria demonstrada pelas plantas, e '100', à morte das plantas (SBCPD, 1995). Também foi avaliada a altura das plantas (desde sua base rente ao colo até ao ápice da folha mais alta, completamente expandida);e ao final de cada estudo, a massa seca da parte aérea. Os resultados foram submetidos à análise de variância, pelo Teste $\mathrm{F}$, e as médias dos tratamentos foram comparadas pelo teste de " $\mathrm{t}$ ", a $5 \%$ de probabilidade.

\section{RESULTADOS E DISCUSSÃO}

No primeiro estudo (2013), para as plantas de açaí (E. oleracea), a aplicação dos herbicidas proporcionou sintomas leves de injúrias às plantas, e estas permaneceram até os $35 \mathrm{DAA}$, sendo que aos 42 DAA os sintomas dissiparam-se completamente, exceto para o setoxydim $\left(184 \mathrm{~g} \mathrm{ha}^{-1}\right)$ e o fomesafen (225 $\mathrm{g} \mathrm{ha}^{-1}$ ) (Tabela 2). Observou-se que os herbicidas sethoxydim (184 $\left.\mathrm{g} \mathrm{ha}^{-1}\right)$, fomesafen $\left(225 \mathrm{~g} \mathrm{ha}^{-1}\right) \mathrm{e}$ MSMA (1.422 $\left.\mathrm{g} \mathrm{ha}^{-1}\right)$ proporcionaram injúrias de até $14 \%$ de fitotoxidade às plantas de E. oleracea. Contudo, aos 49 DAA, as plantas não mostravam mais fitointoxicação.

Para as plantas de juçara (E. edulis), no primeiro estudo (2013), as injúrias ocasionadas pelos tratamentos não superaram os $6 \%$, sendo que as maiores intoxicações foram proporcionadas pelos herbicidas sethoxydim (184 $\left.\mathrm{g} \mathrm{ha}^{-1}\right)$, fomesafen (225 $\left.\mathrm{g} \mathrm{ha}^{-1}\right)$ e MSMA (1.422 $\left.\mathrm{g} \mathrm{ha}^{-1}\right) \mathrm{e}$, novamente ao final do estudo (49 DAA), as folhas já não mostravam mais danos visuais (Tabela 3 ).

Ao comparar-se estes resultados com os de Brancalion et al. (2009), que trabalharam com diversas espécies arbóreas nativas, observou-se que plantas de palmeira-juçara mantidas em tubetes também apresentaram tolerância ao sethoxydim com doses de até $736 \mathrm{~g} \mathrm{ha}^{-1}$, porém dissiparam-se com o decorrer do tempo, o que corrobora os resultados ora encontrados.

Ressalta-se que, no segundo estudo (2014), para ambas as espécies de palmeiras, nenhum dos herbicidas testados ocasionou qualquer sintoma de fitointoxicação, sendo atribuída nota zero de fitotoxidade visual em todas as épocas de avaliação, até ao final do estudo, aos 49 DAA.

Para a altura de plantas de palmeira-açaí no primeiro estudo (Tabela 4), não se verificaram efeitos negativos resultantes da aplicação da maioria dos herbicidas avaliados, com exceção do fluazifop-pbutyl (inibidor da Accase); porém, no primeiro ano, pode ter ocorrido um efeito da escolha da muda, uma vez que no segundo ano não se observou nenhum efeito deste herbicida sobre estes parâmetros avaliados. Apesar de o herbicida fluazifop-p-butyl ter proporcionado menor altura de plantas, nenhum dos herbicidas avaliados interferiu no acúmulo de massa seca das palmeiras (Tabelas 4 e 5).

Já, para a altura de plantas da palmeiraaçaí no segundo estudo (Tabela 5), nenhum dos tratamentos interferiu nesta característica, bem como para sua massa seca.

Com relação à altura de planta para a palmeira-juçara, no primeiro estudo (Tabela 6), nota-se que apenas os herbicidas fluazifopp-butyl (93,8 $\left.\mathrm{g} \mathrm{ha}^{-1}\right)$, quizalofop-p-ethyl $\left(75 \mathrm{~g} \mathrm{ha}^{-1}\right)$ e a mistura clethodim + fenoxapropp-ethyl $\left(50+50 \mathrm{~g} \mathrm{ha}^{-1}\right)$ proporcionaram redução no crescimento das plantas de $18 \%, 24,5 \%$ e $22 \%$, respectivamente, quando comparado com a testemunha, sendo que, para os herbicidas fluazifopp-butyl $\left(93,8 \mathrm{~g} \mathrm{ha}^{-1}\right)$ e quizalofop-p-ethyl (75 g $\left.\mathrm{ha}^{-1}\right)$, ainda houve redução da massa seca da parte aérea de $37 \%$ e $42 \%$, respectivamente. Apesar de os herbicidas fomesafen, lactofen e MSMA não terem interferido na altura de plantas, estes proporcionaram decréscimos no acúmulo de massa seca da parte aérea, de aproximadamente $41 \%, 31 \%$ e $37 \%$, respectivamente, o que pode prejudicar a recomendação destes herbicidas para o palmitojuçara como seletivos, pois a redução da massa seca poderá afetar, também, o diâmetro e a emissão de novas folhas.

No segundo estudo (Tabela 7), para as plantas de palmeira-juçara, nenhum dos tratamentos aplicados apresentou efeito negativo sobre a altura de plantas até ao final do estudo (42 DAA). Ressalta-se que os herbicidas nicosulfuron $\left(50 \mathrm{~g} \mathrm{ha}^{-1}\right)$ e lactofen $\left(168 \mathrm{~g} \mathrm{ha}^{-1}\right)$ proporcionaram decréscimos no acúmulo de massa seca de plantas, porém não significativos. Devese salientar que, no primeiro ano, o herbicida lactofen $\left(168 \mathrm{~g} \mathrm{ha}^{-1}\right)$ reduziu significativamente a biomassa seca de plantas (Tabela 6), o que talvez esteja relacionado com a escolha de mudas. Tal fato traz preocupações quanto à origem da muda, pois poderá afetar o potencial de seletividade deste herbicida. Assim, espécies de interesse florestal podem apresentar respostas distintas aos herbicidas, uma vez que estes indivíduos, mesmo sendo da mesma espécie, podem apresentar alta variabilidade genética devido a sua origem (FERREIRA et al., 2005). 
A maior tolerância da palmeira E. oleracea aos herbicidas testados em relação à palmeira E. edulis, nos dois anos de estudos, pode estar relacionado à maior capacidade de metabolização das moléculas herbicidas, bem como devido a sua agressividade, independentemente de pertencerem ao mesmo gênero, como a presença de perfilhamento em E. oleracea e sua ausência em E. edulis; a menor quantidade, bem como o diâmetro mais estreito de vasos condutores nas folhas de E. oleracea, em comparação a E. edulis, além de maior concentração de corpos silicosos presentes nas células dos vasos condutores de E. oleracea (PEREIRA; QUADROS, 2007). Tais características podem estar relacionadas à maior tolerância aos herbicidas por parte de $E$. oleracea em relação a E. edulis.

Foram observadas diferenças entre as duas espécies de palmeira quanto à sensibilidade aos herbicidas testados, sendo estes responsáveis por quatro diferentes mecanismos de ação nas plantas. Quando ocorreram injúrias visuais, que foram dependentes das moléculas de herbicidas testados, estes dissiparam-se até ao final do estudo, o que pode caracterizar estes herbicidas como tendo bom potencial de seletividade às espécies estudadas.

Contudo, diferenças no acúmulo de biomassa seca foram observadas na palmeira-juçara, mas houve um efeito do ano, que pode estar relacionado à origem do material testado, o que leva a ter-se mais cuidados com esta espécie.

Em culturas perenes, devido ao ciclo ser longo, pode-se exigir métodos e manejos consecutivos para o controle de plantas daninhas até ao momento da colheita. A possibilidade de ter herbicidas com seletividade comprovada sobre espécies de palmeiras permite ao produtor ter uma ferramenta eficaz a mais. O uso de herbicidas com mecanismos de ação diferentes pode auxiliar na rotatividade destes, o que evita a seleção de biótipos de plantas daninhas resistentes, problemas estes que vêm ocorrendo em boa parte de cultivos de interesse agrícola.

TABELA1- Descrição dos tratamentos experimentais. Botucatu-SP, 2013/2014.

\begin{tabular}{lcc}
\hline \multicolumn{1}{c}{ Tratamentos } & $\begin{array}{c}\text { Mecanismo de ação } \\
\text { (inibição) }\end{array}$ & $\begin{array}{c}\text { Dose } \\
\left(\mathrm{g} \mathrm{ha}^{-1}\right)\end{array}$ \\
\hline 1. fluazifop-p-butyl & Accase & 93,8 \\
2. sethoxydim ${ }^{1}$ & Accase & 184,0 \\
3. quizalofop-p-ethyl & Accase & 75,0 \\
4. clethodim + fenoxaprop-p-ethyl & Accase & $50+50$ \\
5. fomesafen & Protox & 225,0 \\
6. lactofen & Protox & 168,0 \\
7. nicosulfuron & ALS & 50,0 \\
8. MSMA & Ácido nucleico & 1422,0 \\
9. testemunha & - & - \\
\hline
\end{tabular}

${ }^{1}$ Adicionou-se 1,0 L ha ${ }^{-1}$ de óleo mineral ASSIST. 
TABELA 2- Porcentagem de fitotoxidade em plantas de Euterpe oleracea(açaí), após a aplicação de diferentes herbicidas. Botucatu-SP, 2013.

\begin{tabular}{|c|c|c|c|c|c|c|c|c|}
\hline \multirow{2}{*}{ Tratamentos } & \multirow{2}{*}{$\begin{array}{l}\text { Doses } \\
\left(\mathrm{g} \mathrm{ha}^{-1}\right)\end{array}$} & \multicolumn{7}{|c|}{ Porcentagem de fitotoxidade (DAA) ${ }^{1}$} \\
\hline & & 7 & 14 & 21 & 28 & 35 & 42 & 49 \\
\hline 1. fluazifop-p-butyl & 93,8 & $0,0 \mathrm{c}$ & $2,5 \mathrm{~b}$ & $2,3 \mathrm{c}$ & $3,8 \mathrm{de}$ & $2,3 \mathrm{~cd}$ & $0,0 \mathrm{c}$ & 0,0 \\
\hline 2. sethoxydim ${ }^{2}$ & 184 & $4,0 \mathrm{ab}$ & $8,3 \mathrm{a}$ & $11,3 \mathrm{a}$ & $14,0 \mathrm{a}$ & $6,5 b$ & $2,3 b$ & 0,0 \\
\hline 3. quizalofop-p-ethyl & 75 & $0,0 \mathrm{c}$ & $2,5 \mathrm{~b}$ & $4,5 \mathrm{bc}$ & $4,0 \mathrm{de}$ & $2,5 \mathrm{~cd}$ & $0,0 \mathrm{c}$ & 0,0 \\
\hline 4. $(\text { cleth. }+ \text { fenox. })^{3}$ & $50+50$ & $0,0 \mathrm{c}$ & $4,3 b$ & $4,8 \mathrm{bc}$ & $5,5 \mathrm{~cd}$ & $3,0 \mathrm{~cd}$ & $0,0 \mathrm{c}$ & 0,0 \\
\hline 5. fomesafen & 225 & $5,0 \mathrm{a}$ & $10,0 \mathrm{a}$ & $10,3 \mathrm{a}$ & $12,0 \mathrm{~b}$ & $8,5 \mathrm{a}$ & $2,8 \mathrm{a}$ & 0,0 \\
\hline 6. lactofen & 168 & $0,0 \mathrm{c}$ & $3,5 b$ & $3,8 \mathrm{c}$ & $3,5 \mathrm{e}$ & $1,8 \mathrm{~d}$ & $0,0 \mathrm{c}$ & 0,0 \\
\hline 7. nicosulfuron & 50 & $0,0 \mathrm{c}$ & $2,8 \mathrm{~b}$ & $3,3 \mathrm{c}$ & $2,8 \mathrm{e}$ & $2,8 \mathrm{~cd}$ & $0,0 \mathrm{c}$ & 0,0 \\
\hline 8. MSMA & 1.422 & $3,0 \mathrm{~b}$ & $8,0 \mathrm{a}$ & $6,5 b$ & $7,0 \mathrm{c}$ & $3,5 \mathrm{c}$ & $0,0 \mathrm{c}$ & 0,0 \\
\hline 9. testemunha & - & - & - & - & - & - & - & - \\
\hline $\mathrm{F}$ tratamento & & $27,43 * *$ & $14,00 * *$ & $14,39 * *$ & $43,26 * *$ & $19,17 * *$ & $86,86^{* *}$ & - \\
\hline C.V. $(\%)$ & & 54,4 & 31,1 & 29,9 & 19,6 & 28,2 & 40,0 & - \\
\hline d.m.s. & & 1,19 & 2,37 & 2,54 & 1,87 & 1,58 & 0,36 & - \\
\hline
\end{tabular}

Médias seguidas de mesma letra, nas colunas, não diferem estatisticamente entre si, pelo teste " $\mathrm{t}$ "( $\mathrm{P} \leq 0,05)$; ${ }^{1} \mathrm{DAA}$ (Dias após a aplicação); ${ }^{2}$ Adicionou-se Assist $\left(1,0 \mathrm{~L} \mathrm{ha}{ }^{-1}\right) ;{ }^{3}$ clethodim + fenoxaprop-p-ethyl.

TABELA 3 -Porcentagem de fitotoxidade em plantas de Euterpe edulis(juçara), após a aplicação de diferentes herbicidas. Botucatu-SP, 2013.

\begin{tabular}{|c|c|c|c|c|c|c|c|c|}
\hline \multirow{2}{*}{ Tratamentos } & \multirow{2}{*}{$\begin{array}{c}\text { Doses } \\
\left(\mathrm{g} \mathrm{ha}^{-1}\right)\end{array}$} & \multicolumn{7}{|c|}{ Porcentagem de fitotoxidade (DAA) ${ }^{1}$} \\
\hline & & 7 & 14 & 21 & 28 & 35 & 42 & 49 \\
\hline 1. fluazifop-p-butyl & 93,8 & 0,0 & $2,5 \mathrm{c}$ & $4,3 \mathrm{~cd}$ & $6,0 \mathrm{c}$ & $3,3 b$ & $0,0 \mathrm{~b}$ & 0,0 \\
\hline 2. sethoxydim ${ }^{2}$ & 184 & 0,0 & $6,0 \mathrm{~b}$ & $9,0 \mathrm{~b}$ & $7,8 \mathrm{abc}$ & $5,3 a$ & $1,8 \mathrm{a}$ & 0,0 \\
\hline 3. quizalofop-p-ethyl & 75 & 0,0 & $2,5 \mathrm{c}$ & $4,8 \mathrm{~cd}$ & $3,3 d$ & $3,0 \mathrm{~b}$ & $0,0 \mathrm{~b}$ & 0,0 \\
\hline 4. $\left(\right.$ cleth. + fenox. $\left.{ }^{3}\right)$ & $50+50$ & 0,0 & $3,0 \mathrm{c}$ & $3,0 \mathrm{~d}$ & $7,0 \mathrm{bc}$ & $3,3 b$ & $0,0 \mathrm{~b}$ & 0,0 \\
\hline 5. fomesafen & 225 & 0,0 & $9,0 \mathrm{a}$ & $13,3 \mathrm{a}$ & $8,3 \mathrm{ab}$ & $5,5 \mathrm{a}$ & $2,8 \mathrm{a}$ & 0,0 \\
\hline 6. lactofen & 168 & 0,0 & $3,0 \mathrm{c}$ & $3,8 \mathrm{~cd}$ & $3,3 \mathrm{~d}$ & $3,0 \mathrm{~b}$ & $0,0 \mathrm{~b}$ & 0,0 \\
\hline 7. nicosulfuron & 50 & 0,0 & $3,0 \mathrm{c}$ & $4,3 \mathrm{~cd}$ & $3,3 d$ & $2,8 b$ & $0,0 \mathrm{~b}$ & 0,0 \\
\hline 8. MSMA & 1.422 & 0,0 & $2,5 \mathrm{c}$ & $6,5 b c$ & $9,3 \mathrm{a}$ & $5,5 \mathrm{a}$ & $2,5 \mathrm{a}$ & 0,0 \\
\hline 9. testemunha & - & - & - & - & - & - & - & - \\
\hline F tratamento & & - & $17,13^{* *}$ & $13,20^{* *}$ & $11,07^{* *}$ & $3,42^{* *}$ & $8,94^{* *}$ & - \\
\hline C.V. $(\%)$ & & - & 28,9 & 31,2 & 24,7 & 34,0 & 94,8 & - \\
\hline d.m.s. & & - & 1,66 & 2,77 & 2,16 & 1,95 & 1,21 & - \\
\hline
\end{tabular}

Médias seguidas de mesma letra, nas colunas, não diferem estatisticamente entre si, pelo teste "t" (P $\leq 0,05)$; ${ }^{1} \mathrm{DAA}$ (Dias após a aplicação); ${ }^{2}$ Adicionou-se Assist $\left(1,0 \mathrm{~L} \mathrm{ha}^{-1}\right) ;^{3}$ clethodim + fenoxaprop-p-ethyl. 
TABELA 4- Efeito de diferentes herbicidas sobre a altura e a massa seca de plantas de Euterpe oleracea(açaí). Botucatu-SP, 2013.

\begin{tabular}{|c|c|c|c|c|c|c|c|c|c|}
\hline \multirow{2}{*}{ Tratamentos } & \multirow{2}{*}{$\begin{array}{c}\text { Doses } \\
\left(\mathrm{g} \mathrm{ha}^{-1}\right)\end{array}$} & \multicolumn{7}{|c|}{ Altura de plantas $(\mathrm{cm})-\left(\mathrm{DAA}^{1}\right)$} & \multirow{2}{*}{$\begin{array}{c}\text { Massa } \\
\text { seca }^{2} \\
(\mathrm{~g})\end{array}$} \\
\hline & & 7 & 14 & 21 & 28 & 35 & 42 & 49 & \\
\hline 1. fluazifop-p-butyl & 93,8 & $45,8 b$ & 47,3 & 47,3 & 46,5 & $46,0 \mathrm{~b}$ & $46,3 b$ & $46,3 b$ & 4,89 \\
\hline 2. sethoxydim ${ }^{3}$ & 184 & $53,8 \mathrm{a}$ & 53,8 & 53,3 & 53,5 & $53,8 \mathrm{ab}$ & $53,5 \mathrm{ab}$ & $53,8 \mathrm{ab}$ & 5,19 \\
\hline 3. quizalofop-p-ethyl & 75 & $51,3 \mathrm{ab}$ & 51,0 & 51,3 & 51,0 & $50,8 \mathrm{ab}$ & $50,5 \mathrm{ab}$ & $50,5 \mathrm{ab}$ & 5,73 \\
\hline 4. $(\text { cleth. }+ \text { fenox. })^{4}$ & $50+50$ & $51,0 \mathrm{ab}$ & 50,8 & 51,3 & 51,0 & $50,8 \mathrm{ab}$ & $51,0 \mathrm{ab}$ & $51,3 \mathrm{ab}$ & 4,53 \\
\hline 5. fomesafen & 225 & $50,3 \mathrm{ab}$ & 49,5 & 50,5 & 50,3 & $50,5 \mathrm{ab}$ & $50,3 \mathrm{ab}$ & $50,3 \mathrm{ab}$ & 5,43 \\
\hline 6. lactofen & 168 & $50,5 \mathrm{ab}$ & 49,8 & 49,5 & 49,8 & $49,5 \mathrm{ab}$ & $49,5 \mathrm{ab}$ & $49,8 \mathrm{ab}$ & 4,74 \\
\hline 7. nicosulfuron & 50 & $48,5 \mathrm{ab}$ & 52,5 & 48,5 & 48,0 & $48,3 \mathrm{ab}$ & $48,3 \mathrm{ab}$ & $48,3 a b$ & 4,40 \\
\hline 8. MSMA & 1.422 & $54,0 \mathrm{a}$ & 48,3 & 53,8 & 52,5 & $53,8 \mathrm{ab}$ & $54,0 \mathrm{ab}$ & $52,0 \mathrm{ab}$ & 6,03 \\
\hline 9. testemunha & - & $53,8 \mathrm{a}$ & 54,0 & 54,3 & 53,8 & $54,8 \mathrm{a}$ & $54,3 \mathrm{a}$ & $54,5 \mathrm{a}$ & 5,95 \\
\hline $\mathrm{F}$ tratamento & & $0,98 *$ & $0,72^{\text {ns }}$ & $0,78^{\mathrm{ns}}$ & $0,76^{\mathrm{ns}}$ & $1,07^{*}$ & $1,01^{*}$ & $0,90 *$ & $0,43^{\text {ns }}$ \\
\hline C.V. $(\%)$ & & 10,7 & 10,9 & 10,6 & 10,9 & 10,8 & 10,6 & 10,6 & 35,8 \\
\hline d.m.s. & & 7,95 & 8,01 & 7,89 & 8,04 & 7,94 & 7,84 & 7,83 & 2,71 \\
\hline
\end{tabular}

Médias seguidas de mesma letra, nas colunas, não diferem estatisticamente entre si, pelo teste " $\mathrm{t}$ " $(\mathrm{P} \leq 0,05) ;{ }^{1} \mathrm{DAA}$ (Dias após a aplicação) ${ }^{2}$ Aos 49 DAA, ${ }^{3}$ adicionou-se Assist $\left(1,0 \mathrm{~L} \mathrm{ha}^{-1}\right) ;{ }^{4}$ clethodim + fenoxaprop-p-ethyl.

TABELA 5 - Efeito de diferentes herbicidas sobre a altura e a massa seca de plantas de Euterpe oleracea(açaí). Botucatu-SP, 2014.

\begin{tabular}{|c|c|c|c|c|c|c|c|c|}
\hline \multirow{2}{*}{ Tratamentos } & \multirow{2}{*}{$\begin{array}{c}\text { Doses } \\
\left(\mathrm{g} \mathrm{ha}^{-1}\right)\end{array}$} & \multicolumn{6}{|c|}{ Altura de plantas $(\mathrm{cm})-\left(\mathrm{DAA}^{1}\right)$} & \multirow{2}{*}{$\begin{array}{c}\text { Massa } \\
\operatorname{seca}^{2}(\mathrm{~g})\end{array}$} \\
\hline & & 7 & 14 & 21 & 28 & 35 & 42 & \\
\hline 1. fluazifop-p-butyl & 93,8 & 50,5 & 50,2 & 50,3 & 50,8 & 52,0 & 53,2 & 6,19 \\
\hline 2. sethoxydim ${ }^{3}$ & 184 & 52,5 & 53,7 & 54,5 & 53,5 & 52,5 & 54,0 & 6,47 \\
\hline 3. quizalofop-p-ethyl & 75 & 38,8 & 42,0 & 42,7 & 42,7 & 42,8 & 43,2 & 4,31 \\
\hline 4. (cleth. + fenox. ${ }^{4}$ ) & $50+50$ & 53,3 & 53,8 & 54,2 & 53,0 & 53,8 & 54,3 & 5,67 \\
\hline 5. fomesafen & 225 & 47,3 & 46,7 & 46,5 & 46,8 & 47,0 & 46,7 & 4,31 \\
\hline 6. lactofen & 168 & 49,7 & 50,0 & 50,3 & 50,0 & 50,3 & 50,5 & 6,85 \\
\hline 7. nicosulfuron & 50 & 51,2 & 52,8 & 53,3 & 53,8 & 53,8 & 53,8 & 6,55 \\
\hline 8. MSMA & 1.422 & 46,8 & 47,0 & 48,0 & 47,7 & 48,5 & 48,2 & 5,35 \\
\hline 9. testemunha & - & 53,0 & 54,3 & 55,7 & 56,2 & 53,33 & 56,5 & 7,07 \\
\hline $\mathrm{F}$ tratamento & & $0,48 \mathrm{~ns}$ & $0,45 \mathrm{~ns}$ & $0,47 \mathrm{~ns}$ & $0,47 \mathrm{~ns}$ & $0,43 \mathrm{~ns}$ & $0,52 \mathrm{~ns}$ & $0,56 \mathrm{~ns}$ \\
\hline C.V. $(\%)$ & & 23,0 & 21,6 & 21,4 & 21,2 & 21,5 & 20,4 & 50,5 \\
\hline d.m.s. & & 19,45 & 18,52 & 18,59 & 18,34 & 18,71 & 17,92 & 4,98 \\
\hline
\end{tabular}

${ }^{1} \mathrm{DAA}$ (Dias após a aplicação), ${ }^{2} \mathrm{Aos} 42 \mathrm{DAA},{ }^{3}$ adicionou-se Assist $\left(1,0 \mathrm{~L} \mathrm{ha}^{-1}\right),{ }^{4}$ clethodim + fenoxaprop-p-ethyl 
TABELA 6- Efeito de diferentes herbicidas sobre a altura e a massa seca de plantas de Euterpe edulis(juçara). Botucatu-SP, 2013.

\begin{tabular}{lccccccccc}
\hline \multirow{2}{*}{ Tratamentos } & $\begin{array}{c}\text { Doses } \\
\left(\mathrm{g} \mathrm{ha}^{-1}\right)\end{array}$ & 7 & 14 & 21 & 28 & 35 & 42 & 49 & Massa \\
\cline { 5 - 9 } seca $^{2}(\mathrm{~g})$
\end{tabular}

Médias seguidas de mesma letra, nas colunas, não diferem estatisticamente entre si, pelo teste " $\mathrm{t}$ " $(\mathrm{P} \leq 0,05)$; ${ }^{1} \mathrm{DAA}(\mathrm{Dias}$ após a aplicação); ${ }^{2}$ Aos 49 DAA; ${ }^{3}$ adicionou-se Assist $\left(1,0 \mathrm{~L} \mathrm{ha}^{-1}\right) ;{ }^{4}$ clethodim + fenoxaprop-p-ethyl.

TABELA 7- Efeito de diferentes herbicidas sobre a altura e a massa seca de plantas de Euterpe edulis(juçara). Botucatu-SP, 2014.

\begin{tabular}{|c|c|c|c|c|c|c|c|c|}
\hline \multirow[b]{2}{*}{ Tratamentos } & \multirow{2}{*}{$\begin{array}{l}\text { Doses } \\
\left(\mathrm{g} \mathrm{ha}^{-1}\right)\end{array}$} & \multicolumn{6}{|c|}{ Altura de plantas $(\mathrm{cm})-\left(\mathrm{DAA}^{1}\right)$} & \multirow{2}{*}{$\begin{array}{c}\text { Massa } \\
\operatorname{seca}^{2}(g)\end{array}$} \\
\hline & & 7 & 14 & 21 & 28 & 35 & 42 & \\
\hline 1. fluazifop-p-butyl & 93,8 & $59,0 \mathrm{ab}$ & $59,0 \mathrm{ab}$ & 59,8 & 60,6 & 60,8 & 60,8 & $6,26 \mathrm{ab}$ \\
\hline 2. sethoxydim ${ }^{3}$ & 184 & $59,0 \mathrm{ab}$ & $59,0 \mathrm{ab}$ & 59,5 & 59,8 & 59,8 & 59,9 & $6,85 \mathrm{ab}$ \\
\hline 3. quizalofop-p-ethyl & 75 & $55,0 \mathrm{ab}$ & $55,4 \mathrm{ab}$ & 56,0 & 56,4 & 57,3 & 57,3 & $8,03 \mathrm{a}$ \\
\hline 4. (cleth. + fenox. $^{4}$ ) & $50+50$ & $59,5 \mathrm{ab}$ & $59,9 \mathrm{ab}$ & 60,3 & 61,6 & 62,0 & 62,0 & $8,03 \mathrm{a}$ \\
\hline 5. fomesafen & 225 & $63,9 \mathrm{ab}$ & $64,0 \mathrm{ab}$ & 65,1 & 65,4 & 65,5 & 65,5 & $8,35 \mathrm{a}$ \\
\hline 6. lactofen & 168 & $65,6 \mathrm{a}$ & $65,4 a$ & 66,0 & 66,3 & 66,0 & 65,6 & $5,35 \mathrm{~b}$ \\
\hline 7. nicosulfuron & 50 & $57,0 \mathrm{ab}$ & $57,6 \mathrm{ab}$ & 58,0 & 58,1 & 58,0 & 57,9 & $5,72 b$ \\
\hline 8. MSMA & 1.422 & $60,3 a b$ & $60,8 \mathrm{ab}$ & 61,8 & 61,8 & 61,8 & 61,9 & $6,22 \mathrm{ab}$ \\
\hline 9. testemunha & - & $52,9 \mathrm{~b}$ & $53,5 \mathrm{~b}$ & 55,5 & 56,6 & 57,9 & 58,8 & $6,67 \mathrm{ab}$ \\
\hline $\mathrm{F}$ tratamento & & $1,06^{*}$ & $0,97^{*}$ & $0,90 \mathrm{~ns}$ & $0,86 \mathrm{~ns}$ & $0,74 \mathrm{~ns}$ & $0,70 \mathrm{~ns}$ & $1,98^{*}$ \\
\hline C.V. $(\%)$ & & 13,1 & 12,9 & 12,7 & 12,4 & 12,1 & 11,9 & 22,5 \\
\hline d.m.s. & & 11,20 & 11,07 & 11,10 & 10,93 & 10,74 & 10,51 & 2,23 \\
\hline
\end{tabular}

Médias seguidas de mesma letra, nas colunas, não diferem estatisticamente entre si, pelo teste " $\mathrm{t}$ " $(\mathrm{P} \leq 0,05) ;{ }^{1} \mathrm{DAA}$ (Dias após a aplicação); ${ }^{2}$ Aos 42 DAA; ${ }^{3}$ adicionou-se Assist $\left(1,0 \mathrm{~L} \mathrm{ha}^{-1}\right) ;{ }^{4}$ clethodim + fenoxaprop-p-ethyl. 


\section{CONCLUSÕES}

Os herbicidas causaram sintomas de fitointoxicação leves a moderados com dissipação total ao final do estudo para ambas as espécies.

Todos os herbicidas testados podem ser recomendados para a palmeira $E$. oleraceae em seu desenvolvimento inicial. Para a palmeira E. edulis, apenas os herbicidas sethoxydim $\left(184 \mathrm{~g} \mathrm{ha}^{-1}\right)$, nicosulfuron $\left(50 \mathrm{~g} \mathrm{ha}^{-1}\right)$ e a mistura clethodim + fenoxaprop-p-ethyl $\left(50+50 \mathrm{~g} \mathrm{ha}^{-1}\right)$ não afetaram seu desenvolvimento inicial.

\section{REFERÊNCIAS}

AGUIAR, T.E.A.; GONÇALVES, C.; PATERNIANI, M.E.A.Z.G.; TUCCI, M.L.S.; CASTRO, C.E.F. Boletim 200: instruções agrícolas para as principais culturas econômicas. 7.ed. Campinas: IAC, 2014. $452 \mathrm{p}$.

BACHEGA, L.P.S.; CARVALHO, L.B.; BIANCO, S.; CECÍLIO FILHO, A.B. Períodos de interferência de plantas daninhas na cultura do quiabo. Planta Daninha, Viçosa, MG, v.31, n. 1, p. 63-70, 2013.

BRANCALION, P.H.S.; ISENHAGEN, I.; MACHADO, R.P.; CHRISTOFFOLETI, P.J.; RODRIGUES, R.R. Seletividade dos herbicidas setoxidim, isoxaflutol e bentazon a espécies arbóreas nativas. Pesquisa Agropecuária Brasileira, Brasília, v.44, p.251-257, 2009.

EMPRESA BRASILEIRA DE PESQUISA AGROPECUÁRIA - EMBRAPA. Centro Nacional de Pesquisa de Solos. Sistema brasileiro de classificação de solos. 2.ed. Rio de Janeiro: EMBRAPA-SPI, 2006. 306p.

FERREIRA, R.A.; DAVIDE, A.C.; ALCÂNTARA, E.N.; MOTTA, M.S. Efeito de herbicidas de préemergência sobre o desenvolvimento inicial de espécies arbóreas. Revista Brasileira de Herbicidas, Maringá, v.4, p. 133-145, 2005.

FREITAS, F.C.L.; GROSSI, J.A.S.; BARROS, A.F.; MESQUITA, E.R.; FERREIRA, F.A. Controle de plantas daninhas na produção de mudas de plantas ornamentais. Planta Daninha, Viçosa, MG, v.25, n.3, p.595-601, 2007.
GUNATHILAKE, H.A.J.; SOMASIRI, L.L.W.; PERIS, T.S.G.; FERNANDO, M.T.N. An appraisal of coconut grower's reaction and observation on coconut research institute recommended cultural practices and other related issues. CRI Report, Cambridge, v.2, n.1, p.89-96, 1993.

MACFADDEN, J. A produção do açaí a partir dos frutos do palmiteiro (Euterpe edulisMartius) na Mata Atlântica. 2005. 100f. Dissertação (Mestrado em Agroecossistemas) - Centro de Ciências Agrárias, Universidade Federal de Santa Catarina, Florianópolis, 2005.

MARTINS, C.C.; BOVI, M.L.A.; SPIERING, S.H. Umedecimento do substrato na emergência e vigor de plântulas de pupunheira. Revista Brasileira de Fruticultura, Jaboticabal, v.31, n.1, p.224-230, 2009a.

MARTINS, C.C.; NAKAGAWA, J.; BOVI, M.L.A. Avaliação da qualidade fisiológica de sementes de açaí. Revista Brasileira de Fruticultura, Jaboticabal, v.31, n.1, p.231-235, 2009b.

MARTINS, D.; TRIGUEIRO, L.R.C.; DOMINGOS, V.D.; MARTINS, C.C.; MARCHI, S. R.; COSTA, N.V. Seletividade de herbicidas aplicados em pós-emergência sobre capim-braquiária. Revista Brasileira de Zootecnia, Viçosa, MG, v.36, p.19691974, 2007.

PEREIRA, M.R.R; MARTINS, D.; RODRIGUES, A.C.P.; SOUZA, G.S.F.; CARDOSO, L. A. Seletividade do herbicida saflufenacil a Eucalyptusurograndis. Planta daninha, Viçosa, MG, v.29, n.3, p.617-624, 2011.

PEREIRA, R.A.; QUADROS, K.E. Euterpe edulis Mart. eE. oleracea Mart. (Arecacea): identificação pela análise de palmitos em conserva. Revista Brasileira de Biociências, Porto Alegre, v.5, supl. 1, p.333-335, 2007.

RAMOS, S.L.F.; MACEDO, J.L.V.; MARTINS, C.C.; LOPES, R.; LOPES, M.T.G. Tratamentos pré-germinativos e procedência de sementes do tucumã-do-amazonas na produção de mudas. Revista Brasileira de Fruticultura, Jaboticabal, v.33, p.962969, 2011. 
RIZZARDI, M.A.; WANDSCHEER, A.C.D. Interferência de Sorghum sudanense e Eleucine indica no cultivo de soja e milho. Planta Daninha, Viçosa, MG, v.32, n. 1, p. 19-30, 2014.

ROMANI; G.N.; SILVA, M.T.; PIVETTA; K.F.L.; PITELLI; R.A.; ALVES; P.L.C. Controle de brilhantina em mudas de Palmeira-açaí. In: CONGRESSO BRASILEIRO DA CIÊNCIA DAS PLANTAS DANINHAS, 27., 2010, Ribeirão Preto. Anais... p.187-191.

SANTOS, A.F. DOS; TESMANN, D.J.; VIDA, J.B.; SANTANA, D.L.Q. Manejo fitossanitário em viveiros de palmeiras para palmito. Colombo: EMBRAPA, Centro Nacional de Pesquisa de Florestas. 2007. 9p. (Circular Técnica, 146).

SBCPD - Sociedade Brasileira da Ciência das Plantas Daninhas. Procedimentos para instalação, avaliação e análise de experimentos com herbicidas. Londrina: SBCPD, 1995. 42 p.
SCHRECKINGER, M.E.; LOTTON, J.; LILA, M.A.; MEJIA, E.G. Berries from SouthAmerica: A comprehensive review on chemistry, health potential, and commercialization. Journal of Medicinal Food, New Rochelle, v.13, n.2, p.233-246, 2010.

SILVA, I.M.; SANTANA, A.C.; REIS, M.S. Análise dos retornos sociais oriundos de adoçãotecnológica na cultura do açaí no Estado do Pará. Amazônia: Ciência \& Desenvolvimento, Belém, v.2, n.3, p.25-37, 2006.

SOUZA, G.S.F.; VITORINO, H.S.; FIOREZE, A.C.L.; PEREIRA, M.R.R.; MARTINS, D. Seletividade de herbicidas na cultura de crambe. Semina. Ciências Agrárias, Londrina, v. 35, p. $161,2014$.

VIEIRA, T.A.; ROSA, L.S.; VASCONCELOS, P.C.S.; SANTOS, M.M.; MODESTO, R.S. Sistemas agroflorestais em áreas de agricultores familiares em Igarapé-Açu, Pará: caracterização florística, implantação e manejo. Acta Amazônica, Manaus, v.37, n. 4, p. 549-558, 2007. 